\title{
CYTOMATRIX, A NEW PROCEDURE TO ENHANCE THE DIAGNOSTIC USEFULNESS OF FINE NEEDLE ASPIRATES
}

\author{
M. Bonucci ${ }^{1,2}$, S. Minelli ${ }^{3}$, C. Lo Castro ${ }^{3}$, C. Camponi ${ }^{1}$, M. Scimeca ${ }^{4}$, A. Scipioni ${ }^{1}$, \\ E. P. Spugnini ${ }^{1}$, A. Baldi ${ }^{1,5}$ \\ ${ }^{1}$ Orchidea LAB S.r.I., Rome, Italy \\ ${ }^{2}$ Associazione Ricerca Terapie Oncologiche Integrate, Rome, Italy \\ ${ }^{3}$ Breast Surgery Service, Villa Stuart Hospital, Rome, Italy \\ ${ }^{4}$ Department of Biomedicine and Prevention, Tor Vergata University of Rome, Rome, Italy \\ ${ }^{5}$ Department of Environmental, Biological and Pharmaceutical Sciences and Technologies, Luigi Vanvitelli University \\ of Campania, Caserta, Italy
}

\section{CORRESPONDING AUTHOR:}

Alfonso Baldi

Orchidea LAB S.r.l., Rome, Italy

Department of Environmental

Biological and Pharmaceutical Sciences and Technologies

Luigi Vanvitelli University of Campania

via Vivaldi 43

81100 Caserta, Italy

E-mail: alfonsobaldi@tiscali.it

ORCID: 0000-0002-8693-3842

Doi: $10.48286 / a r o .2021 .33$

History

Received: May 5, 2021

Accepted: Jun 25, 2021

Published: Sept 1, 2021

\section{ABSTRACT \\ Fine-needle aspirates are still the basis of cytodi- agnosis for neoformation of visceral organs; never- theless, there is an increasing role for ancillary test- ing. Specimens obtained are not always optimal, and prevent a conclusive diagnosis when other complementary tests are needed to reach this goal. This study aims at evaluating a novel technology called "CytoMatrix" to entrap the cytology collec- tions in a synthetic matrix that can be processed as a histology specimen, allowing to perform immu- nohistochemical and molecular biology analyses. Cytological material from twenty-five fine-needle aspirates were collected from different anatomical}

areas and from benign and malignant lesions. The collected aspirated material was transferred onto CytoMatrix, and processed for histology, immunohistochemistry and FISH analyses. In all the cases processed with the synthetic matrix, final diagnosis was reached. Nevertheless, immunohistochemistry and FISH were successfully performed on the malignant neoformations analyzed and the data produced allowed to precisely define the phenotype of the cancer. The results show that this synthetic matrix allows an easy and fast analysis of morphological and molecular characteristics of fine-needle aspirate material from various lesions. 


\section{KEY WORDS}

Fine-needle aspirate; cytology; immunohistochemistry; breast cancer; FISH.

\section{IMPACT STATEMENT}

Definition of an original synthetic matrix that allows an easy and fast analysis of morphological, immunohistochemical and molecular characteristics of fine needle aspirate material from various malignancies.

high ion affinity for cell samples. Chitosan has the capability of efficiently entrapping very small amounts of biological material taken up by needle aspirates, such as single cells or microscopic cell aggregates, inside its three-dimensional structure. The sponge was, then, inserted into a plastic bio-cassette and handled with the steps of classical histological technique. In detail, the aspirated complex CytoMatrix-material was processed as follows: fixation in formalin for at least 12 hours; processing, paraffin embedding and microtome sectioning; application to the sections obtained, of the various diagnostic techniques used in the histopathology laboratory, such histological staining, immunohistochemistry and FISH

\section{Histological staining, immunohistochemistry and FISH}

Paraffin sections obtained from the cytological material entrapped in CytoMatrix were cut at $5 \mu \mathrm{m}$ using a microtome LEICA SM 2000R (Advanced Research Systems Inc., Macungie, PA), dewaxed in xylene, rehydrated through a series of graded ethanol solutions and stained with Gill's Haematoxylin and Eosin (Bio-Optica, Milan). Immunohistochemistry was executed on an automated immunostainer (Bond-III, Leica, Biosystems, Italy), as previously described (10). The primary antibodies used were respectively: Estrogen receptor (clone 6F11), Progesterone receptor (clone 16), ki67 (clone MM1), c-erbB-2 (clone CB11) and E-Cadherin (clone 36B5) (Leica, Biosystems, Italy). Images were obtained by using a light microscope (Microscope Nikon ECLIPSE 55i) equipped with a Digital Image Capture software (Leica Application Suite V4.8). FISH was performed in breast cancers showing $2 / 3+$ HER2 immunohistochemical protein expression using a HER2/CEN 17 dual-color probe kit (ZytoLight Spec, Bremerhaven, Germany). Each slide was observed by using a fluorescence microscope (Eclipse e1000-Nikon) to evaluate the signal of hybridization. A signal ratio of HER2 to chromosome 17 was recorded in a count of a minimum of 50 tumor 
cells and a ratio of $\geq 2$ was regarded as HER2 gene amplification. Breast cancer with HER2 amplification was taken as a positive control.

\section{RESULTS}

Twenty-five fine needle samples were executed on neoformations of various organs, in particular parotid, breast, skin and lymph nodes. Standard hematoxylin and eosin stains were performed, together with analyses by immunohistochemistry and molecular biology studies (FISH). Successively, histological analysis was always performed on tissues obtained either through surgical excision or tru-cut biopsy. Table I summarizes the different samples enrolled in this study, as well as the analyses performed. In detail, immunohistochemistry was carried out on all cases of breast malignancy through E-Cadherin, Estrogen and Progesterone receptors, Ki-67, and Her2-neu antibodies. Finally, in four cases with 2/3 + HER2 immunohistochemical protein staining, also FISH analysis was performed. The Cytomatrix processing method made it possible a final diagnosis in all cases examined. There were no inadequate or doubtful cases and histological analysis on tissues obtained by surgical excision or tru-cut biopsy confirmed the diagnostic data obtained with CytoMatrix. Finally, all the breast cancer samples collected with the synthetic matrix, were appropriate for immunohistochemical and FISH analysis, thus allowing the complete immunophenotypic and molecular description of the tumours. Nevertheless, also for immunohistochemistry and FISH analysis there was full agreement with the data obtained with the classic histological sampling. In figure 1 a paradigmatic example of histological and immunohistochemical staining in a ductal carcinoma performed on paraf-

\begin{tabular}{|c|c|c|c|c|}
\hline SITE & CYTOMATRIX DIAGNOSIS & HISTOLOGICAL DIAGNOSIS & IHC & FISH \\
\hline Sub-cutis & Keratin cyst & Keratin cyst & & \\
\hline Parotid gland & Warthin's tumor & Warthin's tumor & & \\
\hline Parotid gland & Pleomorphic adenoma & Pleomorphic adenoma & & \\
\hline Parotid gland & Pleomorphic adenoma & Pleomorphic adenoma & & \\
\hline Lymph node & Lymphoadenitis & Lymphoadenitis & & \\
\hline Lymph node & Lymphoadenitis & Lymphoadenitis & & \\
\hline Lymph node & Breast cancer metastasis & Breast cancer metastasis & & \\
\hline Lymph node & Breast cancer metastasis & Breast cancer metastasis & & \\
\hline Breast & Ductal carcinoma & Ductal carcinoma & Done & Done \\
\hline Breast & Ductal carcinoma & Ductal carcinoma & Done & Done \\
\hline Breast & Ductal carcinoma & Ductal carcinoma & Done & Done \\
\hline Breast & Ductal carcinoma & Ductal carcinoma & Done & Done \\
\hline Breast & Ductal carcinoma & Ductal carcinoma & Done & \\
\hline Breast & Ductal carcinoma & Ductal carcinoma & Done & \\
\hline Breast & Ductal carcinoma & Ductal carcinoma & Done & \\
\hline Breast & Ductal carcinoma & Ductal carcinoma & Done & \\
\hline Breast & Ductal carcinoma & Ductal carcinoma & Done & \\
\hline Breast & Ductal carcinoma & Ductal carcinoma & Done & \\
\hline Breast & Ductal carcinoma & Ductal carcinoma & Done & \\
\hline Breast & Ductal carcinoma & Ductal carcinoma & Done & \\
\hline Breast & Ductal carcinoma & Ductal carcinoma & Done & \\
\hline Breast & Ductal carcinoma & Ductal carcinoma & Done & \\
\hline Breast & Ductal carcinoma & Ductal carcinoma & Done & \\
\hline Breast & Fibroadenosis & Fibroadenosis & & \\
\hline Breast & Fibroadenosis & Fibroadenosis & & \\
\hline
\end{tabular}

Table I. Characteristics of the patients enrolled and immunohistochemical and FISH data performed. 
fin sections obtained from the cytological material entrapped in CytoMatrix is depicted. In figure 2, the overlapping histological and immunohistochemical results of the same ductal carcinoma after surgical resection are presented. Figure $\mathbf{3}$ shows the results of FISH analysis on four cases with 2/3 + HER2 immunohistochemical protein staining: three out of four cases displayed HER2 amplification. Finally, in figure $\mathbf{4}$ the histological aspect of the material collected by CytoMatrix from a fine needle aspirate of a neoformation of the parotid gland is depicted.

\section{DISCUSSION}

Fine-needle aspiration is considered a not invasive, and easy to perform procedure to diagnose pathologies in various anatomical sites. In the last years with the significant step forwards in the knowledge of the molecular mechanisms causing cancer as well as other pathologies, there has been a significant increase in the requests of biochemical and molecular information in the cytodiagnosis with the final intent to better determine treatment and prognosis (11). Regrettably, cytology specimens, especially once the material has been fixed and stained for the observation at the microscope, often are not suitable for such analyses both for the quality and the quantity of the biological material $(2,3)$. Moreover, performing other cytological samplings may be not possible. Finally, a significant number of ancillary techniques on tissues have been settled to work on material fixed in formalin and paraffin-embedded. This is the case, for example, for immunohistochemistry and FISH (12). In the scientific literature there is a plethora of cell block techniques that have been defined in order to solve these problems. Goal of this article is not to make a comparative analysis between these techniques and CytoMatrix, but to describe the novelty of this procedures and some preliminary data about its reliability.

The use of the synthetic matrix in collecting the cytological sampling allows, on the one hand to recover a significant amount of good quality cytological material and on the other, applies the same methods of histology for the final preparation of the histological preparation. Last but not least, the method allows to recover and observe under the microscope not only single cells, but also microscopic fragments of tissue that preserve, at least in part, the histological structure. This very often facilitates diagnosis. For this reason, we propose the term "micro-histologi- cal diagnosis" for this procedure. It is also necessary to consider that, since the biological material is included in paraffin, it is possible to carry out several consecutive sections, on which to perform different histological staining and/or immunohistochemical and FISH analysis techniques

The Cytomatrix method is easy to apply, is reliable, but above all it can be of great help in those cases in which histological sampling is impractical or not recommended. This method encompasses all these areas: from the simplicity of sampling with a fine needle, to the specificity of the structure representation, to the possible use of the material taken for all further diagnostic investigations (histochemistry, immunohistochemistry, molecular biology). This method can be used not only to make a diagnosis (benign or malignant lesion), which is necessary for an improvement in the diagnostic and therapeutic process, but can be used in cases where histological sampling (as well as surgery) it is not recommended (severe cardiac, hematological and/or vascular pathologies) or impossible due to patient situations (advanced age, severe disability, multi-organ failure). In these cases, a simple sampling with a fine needle will be able to pick up suitable material on which it will be possible not only to make a diagnosis with hematoxylin and eosin staining, but with the material taken it will be possible to perform all the histochemistry, immunohistochemistry and also molecular biology tests. Data produced in this article support the idea that pathologists could include CytoMatrix method, among the various techniques adopted to investigate cytological specimens, to make the cytological material appropriate not only to morphological analysis, but also to immunohistochemical and molecular analysis. Further experimentations are ongoing to demonstrate the suitability of the cytological material collected with this technique for more sophisticated assays of molecular biology, such as mutational analysis and next generation sequencing.

\section{ACKOWLEDGEMENTS}

The authors thank Dr. Antonio Santoro (UCS Diagnostics, Rome, Italy) for kindly providing the synthetic matrix "Cytomatrix" for the study. We wish also to thank dr. Francesca Cimirro for her precious support in the organization and coordination of Orchidealab. 

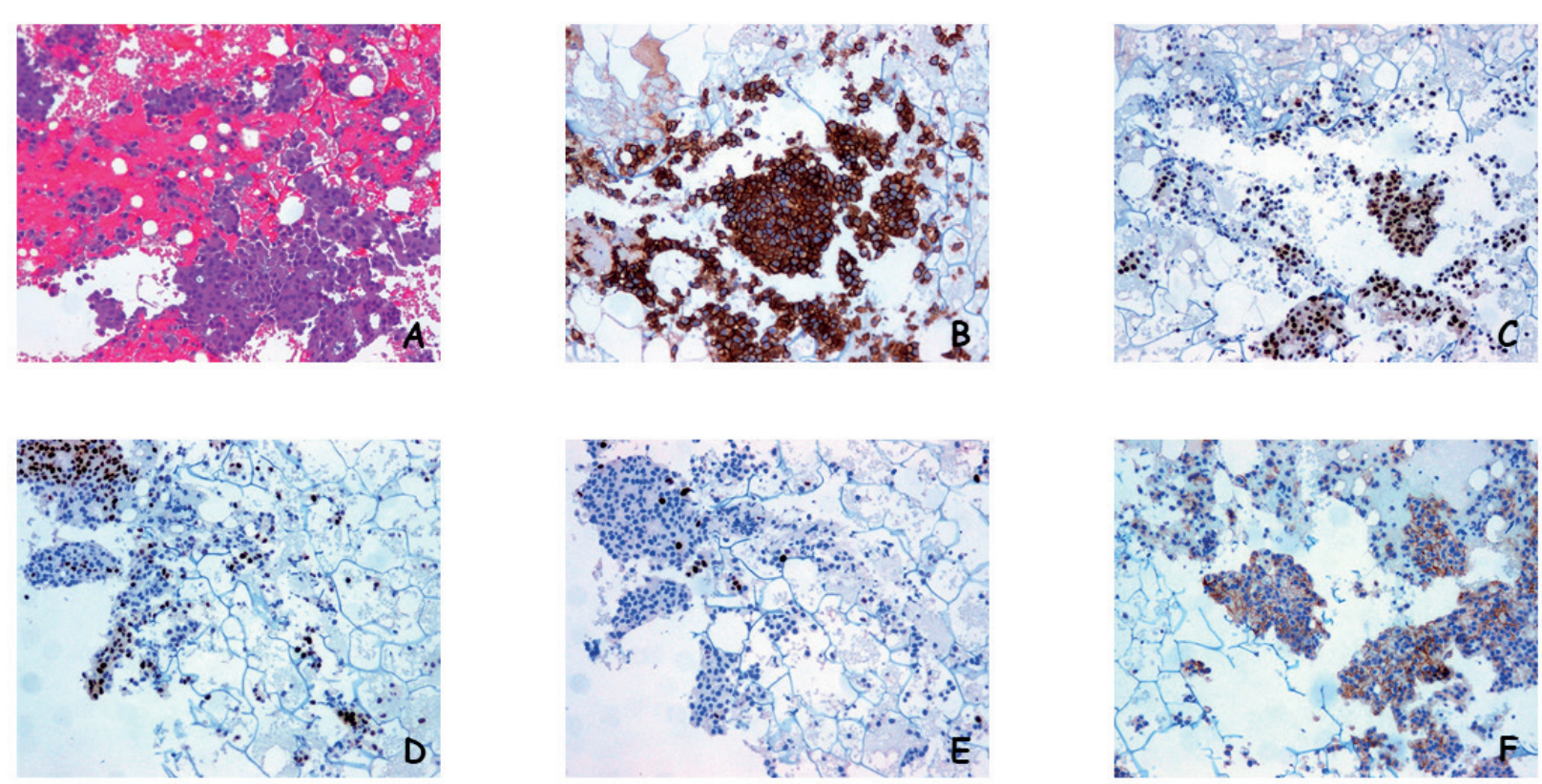

Figure 1. Histological and immunohistochemical pattern of the material collected by Cytomatrix from a fine needle aspirate of a lump of the breast. a. Histological analysis allowed the final diagnosis of ductal carcinoma. To note, the material stained with eosin is the remaining of the synthetic matrix (H\&E, original magnification X10). b. Immunohistochemical analysis showed high expression of E-Cadherin (ABC, original magnification X10). c. The cancer displayed high expression of Estrogen receptor (ABC, original magnification X10). d. Expression pattern of Progesterone receptor $(A B C$, original magnification X10). e. Expression pattern of the proliferation marker ki67 in cancer cells (ABC, original magnification X10). $f$. High expression of HER2-neu on the cytoplasmic membrane of cancer cells (ABC, original magnification X10).
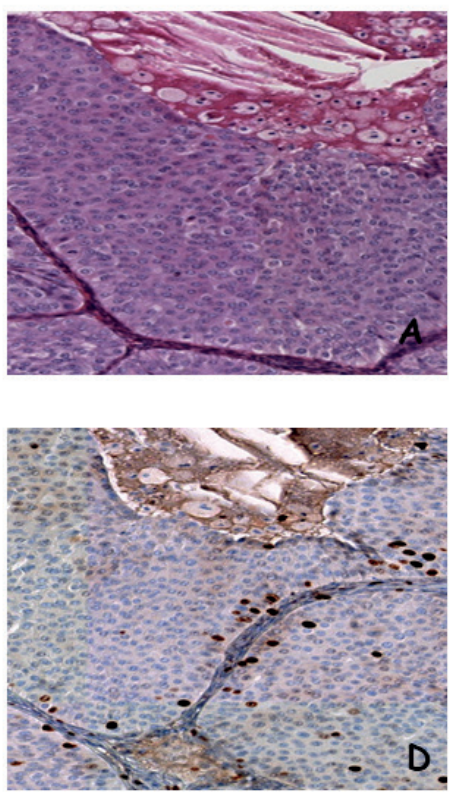
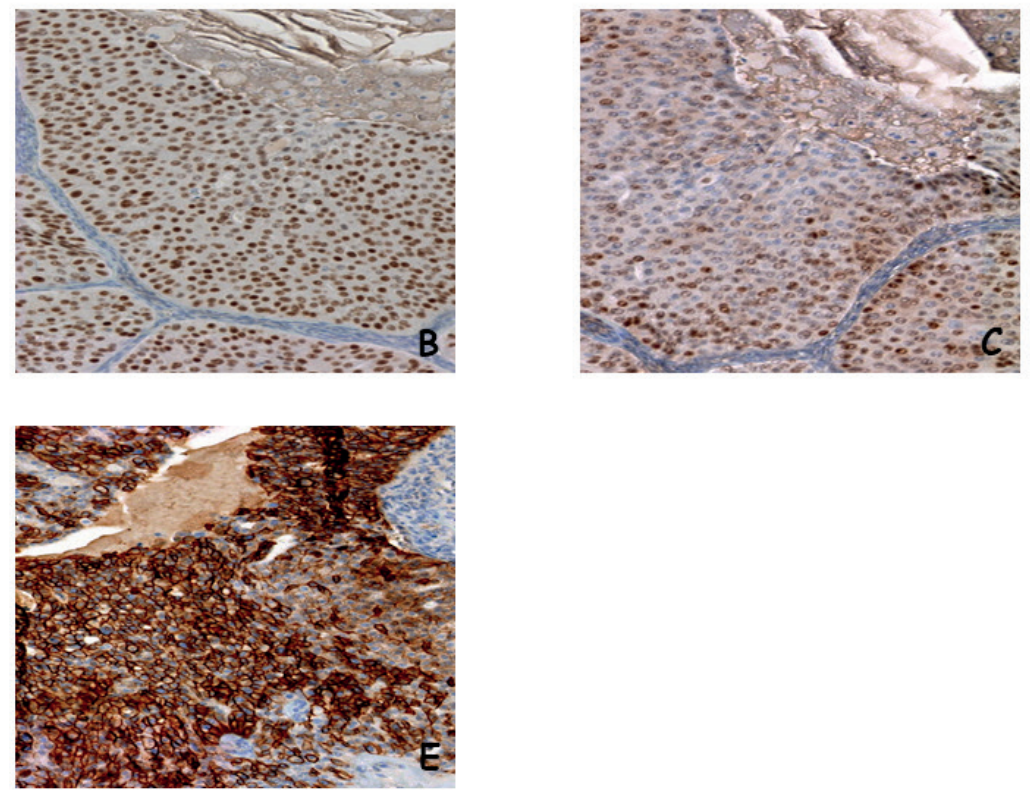

Figure 2. The histological and immunohistochemical pattern of the same carcinoma presented in figure 1 are overlapping with the data produced with Cytomatrix. $\boldsymbol{a}$. Histological analysis confirmed the diagnosis of ductal carcinoma (H\&E, original magnification X10). $\boldsymbol{b}$. The cancer displayed high expression of Estrogen receptor (ABC, original magnification X10). c. Expression pattern of Progesterone receptor (ABC, original magnification X10). $\boldsymbol{d}$. Expression pattern of the proliferation marker ki67 in cancer cells (ABC, original magnification X10). e. High expression of HER2-neu on the cytoplasmic membrane of cancer cells ( $A B C$, original magnification X10). 

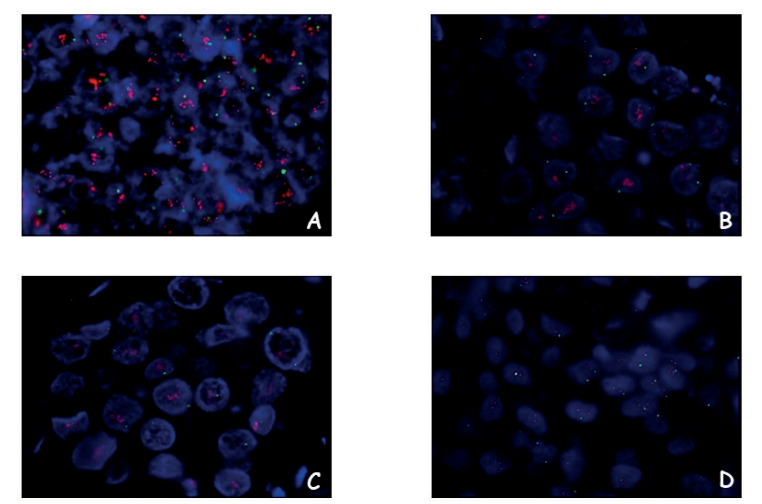

Figure 3. FISH analysis for HER2-neu of the material collected by CytoMatrix from a fine needle aspirate of two ductal carcinomas showing 2/3+ HER2 immunohistochemical protein expression. $\boldsymbol{a}$. In this breast cancer specimen, the signal ratio of HER2 $\geq 2$ was regarded as HER2-neu gene amplification. $\boldsymbol{b}$. In this breast cancer specimen, the signal ratio of HER2 $\geq 2$ was regarded as HER2-neu gene amplification. c. In this breast cancer specimen, the signal ratio of HER2 $\geq 2$ was regarded as HER2-neu gene amplification. $\boldsymbol{d}$. In this breast cancer specimen, the signal ratio of HER2 $<2$ was regarded as no HER2-neu gene amplification.

\section{ETHICS}

\section{Fundings}

There were no institutional or private fundings for this article.

\section{Conflict of interests}

The authors have declared no conflict of interests.

\section{Availability of data and material}

All data generated or analysed during this study are included in this published article.

\section{Code availability}

N/A

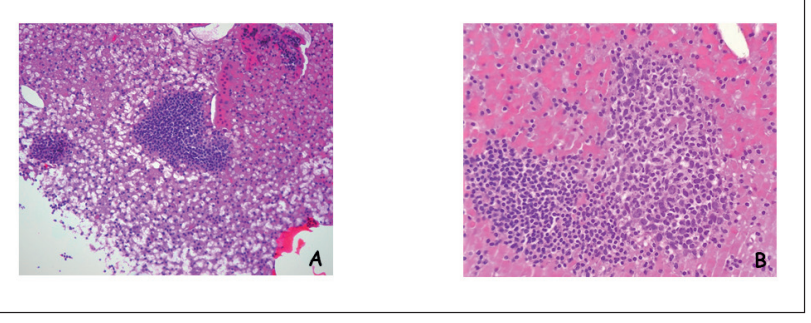

Figure 4. Histological aspect of the material collected by CytoMatrix from a fine needle aspirate of a neoformation of the parotid gland. $\boldsymbol{a}$. Histological analysis allowed the final diagnosis of Whartin's tumour, based on the recognition of the two epithelial and lymphoid tissue components (H\&E, original magnification X10). b. Higher magnification better showing the two different population of cells characteristic of Warthin's tumour (H\&E, original magnification X20).

\section{Authors' contribution}

$M B$ and $A B$ performed the histopathological analyses, analyzed the data and wrote the manuscript, SM and CLC performed the fine-needle aspirates, CC and AS performed the histopathological and immunohistochemical procedures, MS performed the FISH procedures, EPS contributed in the interpretation of the data.

\section{Ethical approval}

The study protocol was approved in accordance with the ethical standards established in the Declaration of Helsinki of 1946 (World Medical Association. World Medical Association Declaration of Helsinki: ethical principles for medical research involving human subjects. JAMA 2013 Nov 27;310(20):2191-4. Doi: 10.1001/ jama.2013.281053).

\section{Consent to participate}

Written informed consent was obtained from all the subjects before the collection of the fine needle samples with CytoMatrix. 


\section{REFERENCES}

1. Aisner DL, Sams SB. The role of cytology specimens in molecular testing of solid tumours: techniques, limitations, and opportunities. Diagn Cytopathol 2012;40:511-24.

2. Kang A, Miranda A, de Boer B. Manufactured Cell Blocks: Turning Smears into Sections. Acta Cytologica 2019;63:28-34.

3. Fowler LJ, Lachar WA. Application of Immunohistochemistry to Cytology. Arch Pathol Lab Med 2008; 132:373-83.

4. Gong Y, Joseph T, Sneige N. Validation of commonly used immunostains on cell-transferred cytologic specimens. Cancer 2005;105:58-64.

5. da Cunha Santos G, Saieg MA, Troncone G, Zeppa P. Cytological preparations for molecular analysis: A review of technical procedures, advantages and limitations for referring samples for testing. Cytopathology 2018;29:125-32.

6. Sinchita Roy-Chowdhuri. Molecular testing of residual cytology samples: Rethink, reclaim, repurpose. Cancer Cytopathol 2019; 127:15-7.

7. Spugnini EP, Menicagli F, Giaconella R, et al. Filling the gap between histology and cytology: description of an innovative technology (Cy- tomatrix) to increase the diagnostic effectiveness of fine needle aspirates data. J Clin Pathol 2020;jclinpath-2020-206545.

8. Bruschini S, di Martino S, Pisanu ME, et al. CytoMatrix for a reliable and simple characterization of lung cancer stem cells from malignant pleural effusions. J Cell Physiol 2020;235:1877-87.

9. Scarpino S, Taccogna S, Pepe G, et al. Morphological and Molecular Assessment in Thyroid Cytology Using Cell-Capturing Scaffolds. Horm Metab Res May 11, 2020. Online ahead of print.

10. Trecca A, Ortica F, Marinozzi G, Borghini R, Camponi C, Baldi A. Gastrointestinal stromal tumor with skeinoid fibers: an unusual presentation. Tech Coloproctol 2018;22:895-7.

11. Paul A VanderLaan. Molecular markers: Implications for cytopathology and specimen collection. Cancer Cytopathol 2015;123:454-60.

12. Howard H Wu, Kelly J Jones, Harvey M Cramer. Immunocytochemistry performed on the cell-transferred direct smears of the fine-needle aspirates: a comparison study with the corresponding formalin-fixed paraffin-embedded tissue. Am J Clin Pathol 2013;139:754-8. 